\title{
A QUESTION OF C. R. HOBBY ON REGULAR $p$-GROUPS
}

\author{
by I. D. MACDONALD \\ (Received 14th January 1972)
}

In (2) a finite $p$-group $G$ is said to be nearly regular if it has the following two properties:

(i) There exists a central subgroup $Z$ of order $p$ and $G / Z$ is regular.

(ii) If $x \in G$ and $y \in \gamma_{2}(G)$, then $g p\{x, y\}$ is regular.

(For unfamiliar notation we refer to (1).) C. R. Hobby proved in (2) that (i) implies (ii) when $p=2$ or 3 , and an open question is whether (i) implies (ii) for $p \geqq 5$. It has been suggested in (3) and (4) that this is a deep problem, comparable to the Hughes problem perhaps, though the solution is in fact quite simple; it seems worth while to set the record straight with the present note. We shall exhibit, for any $p \geqq 5$, a finite metabelian p-group with (i) but not (ii).

In considering the structure of a metabelian $p$-group $G$ with property (i) but not (ii) we shall be guided by the necessary and sufficient conditions given in (1) for a nilpotent metabelian $p$-group to be regular, namely that $\gamma_{p}(H) \leqq \gamma_{2}(H)^{p}$ for every two-generator subgroup $H$. Such a group has of course $p \geqq 5$ and $G / Z$ will not have exponent $p$.

Let us suppose that $G$ is generated by $\{a, b\}$ and that $G$ has class $p+1$, more precisely that $Z=\gamma_{p+1}(G)$. It follows according to (i) that

$$
\gamma_{p}(G) \leqq \gamma_{2}(G)^{p} Z
$$

which implies that $\gamma_{p+1}(G) \leqq \gamma_{3}(G)^{p}$, and so $\gamma_{p}(G) \leqq \gamma_{2}(G)^{p}$. Let us define the subgroup $K$ as $g p\{x, y\}$, where $x=a$ and $y=[a, b] ; K$ is to be the non-regular subgroup appearing in (ii). Let us make $K$ non-regular by arranging that $\gamma_{p}(K) \leqq \gamma_{2}(K)^{p}$ is false. We have $\gamma_{p}(K) \leqq \gamma_{p+1}(G) \leqq \gamma_{3}(G)^{p}$ and so we must not allow $\gamma_{3}(G)^{p} \leqq \gamma_{2}(K)^{p}$; in particular $\gamma_{3}(G)^{p} \neq 1$. Since $G / Z$ is regular we have $\gamma_{p}(K) \leqq \gamma_{2}(K)^{p} Z$, however, and the fact that $Z$ has order $p$ now implies that $Z \leqq K$. Note that $\gamma_{p}(K)$ cannot be 1 as $K$ is non-regular, so it seems reasonable to put $Z=g p\{z\}$, where $z=[a, b,(p-1) a]$.

We return to $\gamma_{3}(G)^{p} \pm \gamma_{2}(K)^{p}$. Since

$$
\gamma_{2}(K)^{p}=g p\left\{[a, b, i a]^{p}: 1 \leqq i<p\right\}
$$

we shall aim to have $[a, b, b]^{p} \notin \gamma_{2}(K)^{p}$. Earlier remarks indicate that we must avoid $z \in \gamma_{2}(K)^{p}$. Since $\gamma_{p}(G) \leqq \gamma_{2}(G)^{p}$ we face the problem of specifying $[a, b, i a,(p-2-i) b]$ as an element of $\gamma_{2}(G)^{p}$, for $0 \leqq i \leqq p-2$.

We put

$$
[a, b,(p-2) a]=[a, b, b]^{p} z,
$$

a relation which implies $z=[a, b, a, b]^{p}$ and therefore $[a, b,(p-2) a] \in \gamma_{2}(G)^{p}$, 
without obviously entailing $z \in \gamma_{2}(K)^{p}$. We note the further consequence

Next we put

$$
[a, b, a, b]^{p^{2}}=1 \text {. }
$$

$$
[a, b, b, b]=[a, b, a, a, b]=1
$$

and this trivially yields $[a, b, i a,(p-2-i) b]=1$ for $0 \leqq i<p-2$. Such relations as we have mentioned do not imply that $G$ is a $p$-group, and so we put $a^{p^{2}}=b^{p^{2}}=1$.

If $H$ is a proper subgroup of $G$ and if $H$ has 2 generators, then the relations give the fact that, modulo $Z, H$ has class $p-1$; so $G / Z$ is regular by the criterion of (1), and we have (i), if $Z$ has order $p$. It therefore remains to establish that $z \neq 1$ and that $[a, b, b]^{p} \notin \gamma_{2}(K)^{p}$, in order to prove (i) and disprove (ii).

At this point a construction, which we shall merely outline, is called for. We start with symbols $c_{00}, c_{10}, \ldots, c_{p-1,0}, c_{01}, c_{11}$ which we suppose generate an abelian group of exponent $p^{2}$, and we impose the further relations

$$
\begin{aligned}
& c_{20}^{p}=c_{30}^{p}=\ldots=c_{p-1, o}=1, \\
& c_{p-2,0}=c_{01}^{p} c_{11}^{p}, c_{p-1,0}=c_{11}^{p} .
\end{aligned}
$$

There results a group of order $p^{p+4}$. From this we may obtain the required example $G$ by adjoining elements $a$ and $b$, using extension theory, so that

$$
\begin{aligned}
& a^{p^{2}}=b^{p^{2}}=1, \quad[a, b]=c_{00}, \\
& {\left[c_{i j}, a\right]=c_{i+1, j},\left[c_{i j}, b\right]=c_{i, j+1},}
\end{aligned}
$$

where $c_{i+1, j}$ and $c_{i, j+1}$ are 1 if not in the initial set of symbols. Then $G$ will have order $p^{p+8}$. Once this is established it is clear that $z=c_{p-1,0}$ has order $p$ and that $[a, b, b]^{p}=c_{01}^{p} \notin \gamma_{2}(K)^{p}$ where $K=g p\left\{a, c_{00}\right\}$ and $\gamma_{2}(K)^{p}=g p\left\{c_{10}^{p}\right\}$. Hence:

Theorem. There is a metabelian p-group, for each $p \geqq 5$, that satisfies (i) and does not satisfy (ii).

This group does not satisfy the conclusion of Hobby's theorem in (2) about nearly regular $p$-groups either; a fact which may be verified directly by means of Corollary 2.3 of (1) for instance.

\section{REFERENCES}

(1) WaRREN BrisLeY and I. D. MACDonald, Two classes of metabelian p-groups, Math. Z. 112 (1969), 5-12.

(2) C. R. Hовву, Nearly regular p-groups, Canad. J. Math. 19 (1967), 520-522.

(3) I. D. Macdonald, The Hughes problem and others, J. Austral. Math. Soc. 10 (1969), 475-479.

(4) I. D. MaCDonald, Solution of the Hughes problem for finite $p$-groups of class 2p-2, Proc. Amer. Math. Soc. 27 (1971), 39-42.

Department of Mathematics

THE UNIVERSITY OF STIRLING

STIRLING, SCOTLAND 